\title{
Stability of the perturbative vacuum against spatial variations of the Polyakov loop
}

\author{
Kenji Fukushimat and Koichi Ohtat \\ Institute of Physics, University of Tokyo, 3-8-1 Komaba, Meguro-ku, Tokyo 153-8902, Japan
}

\begin{abstract}
We investigate the effective action of the Polyakov loop with spatial variations. We expand the effective action not in powers of derivatives or momenta, but in powers of variational amplitudes. At one-loop order the results suggest that the instability towards the confining vacuum may be caused by the variational terms.

11.10.Wx, 12.38.Aw
\end{abstract}

\section{INTRODUCTION}

It is believed that the vacuum of the SU(N) Yang-Mills theories should be in the colour-confined phase at zero or low temperature, while at sufficiently high temperature the colour-deconfined phase would emerge where perturbative calculations do well or at least somewhat make sense.

In the $\mathrm{SU}(N)$ pure Yang-Mills theories at finite temperature the centre symmetry is known to be deeply connected with the criteria of colour confinement [1, 22,3. The order parameter of the symmetry is given by the expectation value of the Polyakov loop operator,

$$
\Omega=\frac{1}{V} \int \mathrm{d}^{3} x\langle\Omega(\boldsymbol{x})\rangle=\frac{1}{V} \int \mathrm{d}^{3} x\left\langle\frac{1}{N} \operatorname{tr} \mathcal{T} \exp \left(-\mathrm{i} \int_{0}^{\beta} \mathrm{d} x_{4} A_{4}\left(x_{4}, \boldsymbol{x}\right)\right)\right\rangle .
$$

Here $\beta=1 / T$ is the inverse temperature, $\mathcal{T}$ is the time ordering operator and tr denotes the trace over the colour index in the fundamental representation. One can readily relate it to the free energy $F_{q}$ in the presence of a single quark with infinitely heavy mass: $\Omega=\exp \left(-\beta F_{q}\right)$. Therefore, the confined phase $\left(F_{q}=\infty\right)$ corresponds to $\Omega=0$, whereas $\Omega \neq 0$ in the deconfined phase. If the vacuum is centre symmetric, that is to say $\Omega=L \Omega(L \in \mathrm{Z}(N))$, it follows that $\Omega=0$, i.e. that the realization of the centre symmetry is accompanied by the confining vacuum.

The effective potential of the Polyakov loop has been calculated both in the perturbative approach [4,5,6,6,8] and in the strong-coupling expansion on the lattice [9, 10]. The perturbative results have revealed that the centre symmetry is broken spontaneously due to the appearance of the Debye mass at high temperature. It was also proven that any perturbative calculation inevitably leads to $\Omega=1$, that is the perturbative vacuum 11], though the proof is based on the suspicious assumption that the Haar measure could be harmlessly negligible. Recently, however, Boháčik 12] and Engelhardt and Reinhardt [13] have pointed out in the Polyakov gauge (static gauge $\partial_{4} A_{4}=0$ with an additional condition that $A_{4}$ should be diagonal) that the effective action, which includes not only potential terms but also kinetic terms, has a singularity right at the perturbative vacuum. The singularity stems from the Haar measure, whose meaning is still a little confused 114,15,16. This fact seems to suggest that the Haar measure or some configurations with spatial variations such as calorons (multi-instantons at finite temperature), magnetic monopoles and domain walls might be relevant to the dynamics of the confinement-deconfinement mechanism.

These former results, however, are somewhat ambiguous with respect to the two points, i.e. renormalization and gauge invariance. Because of the singular character of the Polyakov gauge, it is likely that gauge spurious objects might arise from incomplete renormalization. Thus instead of using the singular Polyakov gauge we prefer the covariant background field gauge in our calculation as in [7, 8 at the cost of the renormalization necessary for the Polyakov loop.

One might think that the kinetic energy must be dominated by the tree term and that the loop corrections should be much smaller than the tree term so as to make sense of the perturbative calculation. However, the longitudinal gluon propagator, for instance, acquires the Debye mass from the contribution of the loop corrections and then in the soft momentum region the tree term $\sim(\partial A)^{2}$ proportional to squared momentum can be certainly negligible compared with the finite Debye mass. Of course, when the momentum becomes larger the tree term grows to be dominant eventually. Consequently, one has to take account of loop corrections until the momentum exceeds the

${ }^{*}$ E-mail: fuku@nt1.c.u-tokyo.ac.jp

${ }^{\dagger}$ E-mail: ohta@nt1.c.u-tokyo.ac.jp 
order of $T$. Because we are interested in the momentum region around $T$, the effective action should be expanded in powers of variational amplitudes instead of derivatives or momenta [7, 13$]$.

The outline of this paper is as follows. We define the effective action of the Polyakov loop in Sec. II. Using the same technique as the derivation of the gauge dependence identity, we show that the effective action of the Polyakov loop is generally gauge invariant even if the classical fields do not satisfy the on-shell conditions (the equations of motion). Sec. III is divided into three subsections. Firstly, we expand the one-loop effective action to quadratic variational terms. The result suggests possible instability for the perturbative vacuum. Secondly, quartic terms are found to stabilize the slipped vacuum. Finally we remark upon the zero temporal modes and the resultant cubic term in the potential. In Sec. IV the obtained effective action is examined numerically to give rise to the vacuum instability. We conclude our discussion and comment upon problems inherent in our result in Sec. $\mathrm{V}$.

\section{DEFINITION OF THE EFFECTIVE ACTION}

The effective action in terms of gauge fields $A_{\mu}^{a}$ is defined in a field-theoretic manner by the equation

$$
\exp (\Gamma[\bar{A}])=\int \mathcal{D} A \mathcal{D} \bar{c} \mathcal{D} c \exp \left(S[c, \bar{c}, A]+S_{\mathrm{src}}\right)
$$

where the action $S$ is the sum of the classical action $S_{\mathrm{cl}}$, the gauge-fixing action $S_{\mathrm{gf}}$ and the ghost action $S_{\text {ghost }}$, namely,

$$
\begin{aligned}
S[c, \bar{c}, A] & =S_{\mathrm{cl}}[A]+S_{\mathrm{gf}}[A]+S_{\text {ghost }}[c, \bar{c}, A] \\
& =\int^{\beta} \mathrm{d}^{4} x\left\{-\frac{1}{4 g^{2}} F_{\mu \nu a} F_{\mu \nu}^{a}-\frac{1}{2 g^{2} \xi} \mathcal{F}_{a} \mathcal{F}^{a}-\bar{c}_{a} \frac{\delta \mathcal{F}^{a}}{\delta A_{\mu}^{b}} D_{\mu} c^{b}\right\}
\end{aligned}
$$

and the source action $S_{\mathrm{src}}$, which only removes the one-particle reducible diagrams, is written as

$$
S_{\mathrm{src}}=\int^{\beta} \mathrm{d}^{4} x J_{\mu a}\left(A_{\mu}^{a}-\bar{A}_{\mu}^{a}\right) \quad J_{\mu a}=-\frac{\delta \Gamma[\bar{A}]}{\delta \bar{A}_{\mu}^{a}} .
$$

Here $\xi$ is the gauge parameter and $\mathcal{F}^{a}$ is to be chosen appropriately to fix a gauge. The upper and lower indices of colour should be distinguished, as we employ the ladder basis later (the Cartan metric in the ladder basis is not diagonal). For the purpose of defining the effective action of the Polyakov loop, we replace a part of the degrees of freedom, that is described by the elementary fields, with the (extended) Polyakov loops defined as

$$
\Omega^{n}(\boldsymbol{x})=\frac{1}{N} \operatorname{tr}\left\{\mathcal{T} \exp \left(-\mathrm{i} \int_{0}^{\beta} \mathrm{d} x_{4} A_{4}\left(x_{4}, \boldsymbol{x}\right)\right)\right\}^{n}
$$

where $n$ runs over $1,2, \ldots, N-1$ corresponding to the Cartan subalgebra (the neutral gluons), or alternatively $1,2, \ldots, N$ with a constraint to force the untraced Polyakov loops to be Hermitian. Because the Polyakov loops are static and written only by the gauge potential $A_{4}$, it is natural to transfer the variables from $\tilde{A}_{4}^{n}(\boldsymbol{x})$ to $\Omega^{n}(\boldsymbol{x})$, where

$$
\tilde{A}_{4}^{n}(\boldsymbol{x})=\frac{1}{\beta} \int_{0}^{\beta} \mathrm{d} x_{4} A_{4}^{n}\left(x_{4}, \boldsymbol{x}\right) .
$$

Then the source action (4) can be replaced by

$$
\begin{aligned}
S_{\mathrm{src}} & =\int^{\beta} \mathrm{d}^{4} x\left\{\tilde{J}_{\mu a}\left(A_{\mu}^{a}-\bar{A}_{\mu}^{a}\right)+K_{n}\left(\Omega^{n}-\bar{\Omega}^{n}\right)\right\} \\
\tilde{J}_{\mu a} & =-\frac{\delta \Gamma[\bar{A}, \bar{\Omega}]}{\delta \bar{A}_{\mu}^{a}} \quad\left(\int_{0}^{\beta} \mathrm{d} x_{4} \tilde{J}_{4 n}\left(x_{4}, \boldsymbol{x}\right)=0\right) \\
K_{n} & =-\frac{\delta \Gamma[\bar{A}, \bar{\Omega}]}{\delta \bar{\Omega}^{n}}
\end{aligned}
$$

where the source $\tilde{J}_{\mu a}$ is almost the same as that in the source action (ब) except that no more $\tilde{A}_{4}^{n}$ arises out of it. The expression in the brackets represents this constraint on $\tilde{J}_{\mu a}$. The source for $\tilde{A}_{4}^{n}$ is not $\tilde{J}_{\mu a}$ but $K_{n}$, in which the 
Polyakov loops are regarded as the dynamical variables in place of $\tilde{A}_{4}^{n}$. Then setting $\bar{A}=0$ we define the effective action of the Polyakov loops that is denoted as $\Gamma[\bar{\Omega}]$.

In the same manner as the derivation of the gauge dependence identity [17], it is easy to show that the effective action defined above is completely independent of a gauge choice of either $\mathcal{F}$ or $\xi$. The infinitesimal change of the gauge-fixing condition,

$$
\mathcal{F}^{a}[A] \rightarrow \mathcal{F}^{a}[A]+\delta \mathcal{F}^{a}[A] \quad \xi \rightarrow \xi+\delta \xi
$$

induces the variation only for the gauge-fixing action as the following:

$$
\begin{aligned}
\delta S_{\mathrm{gf}}[A] & =-\int^{\beta} \mathrm{d}^{4} x\left(\frac{1}{\xi} \mathcal{F}_{a} \delta \mathcal{F}^{a}-\frac{\delta \xi}{2 \xi^{2}} \mathcal{F}_{a} \mathcal{F}^{a}\right) \\
& =-\int^{\beta} \mathrm{d}^{4} x \frac{1}{\xi} \mathcal{F}_{a} \delta^{\prime} \mathcal{F}^{a} \\
& =-\int^{\beta} \mathrm{d}^{4} x \mathrm{~d}^{4} y \frac{\delta S[A]}{\delta A_{\mu}^{a}(x)}\left(D_{\mu}\right)_{b}^{a}(x) \mathcal{G}_{c}^{b}(x-y) \delta^{\prime} \mathcal{F}^{c}(y)
\end{aligned}
$$

where $\mathcal{G}$ is the full propagator of the ghost fields defined by the equation of motion,

$$
\int^{\beta} \mathrm{d}^{4} y \frac{\delta \mathcal{F}^{a}(x)}{\delta A_{\mu}^{b}(y)}\left(D_{\mu}\right)_{c}^{b}(y) \mathcal{G}_{d}^{c}(y-z)=-\delta_{d}^{a} \delta(x-z) .
$$

It is the essential point that $\delta S_{\mathrm{gf}}$ can be compensated by an appropriate gauge transformation,

$$
\delta A_{\mu}^{a}(x)=\left(D_{\mu}\right)_{b}^{a}(x) \int^{\beta} \mathrm{d}^{4} y \mathcal{G}_{c}^{b}(x-y) \delta^{\prime} \mathcal{F}^{c}(y) .
$$

The only terms varied under the subsequent transformations (8) and (11) are the source terms, to say more precisely, fields coupled with the source, provided that the integration measure contains the factor $1 / \sqrt{\xi}$ [17]. In the present case the source terms are given by (目) among which one term with $\tilde{J}_{\mu a}$ vanishes because we have set $\bar{A}=0$. In the other terms with $K_{n}$, the coupled quantum fields transformed by (11) are the Polyakov loops $\Omega^{n}$. They are definitely invariant for any periodic gauge transformation. Therefore, it is obvious that the effective action of the Polyakov loops does not depend on a gauge choice at all due to the gauge invariance of the Polyakov loops even when the classical fields $\bar{\Omega}^{n}$ do not satisfy the stationary condition.

For the purpose of the perturbative calculation it is convenient to expand the source term (『) in powers of the elementary gauge fields. We parameterize the classical Polyakov loops as

$$
\bar{\Omega}^{n}=\frac{1}{N} \operatorname{tre}{ }^{-\mathrm{i} n \beta a}=\frac{1}{N} \sum_{i=1}^{N} \mathrm{e}^{-\mathrm{i} n \beta a_{i}}
$$

with the Hermitian constraint,

$$
\sum_{i=1}^{N} a_{i}=0 .
$$

Later on we will make use of the background field method with the background fields $a=\operatorname{diag}\left(a_{1}, a_{2}, \ldots, a_{N}\right)$ introduced in (12). It is important to note that this definition of the background fields contains the renormalization of the Polyakov loops from the beginning [6]. The gauge fields are decomposed into the quantum parts and the background parts respectively,

$$
A_{\mu}(x)=\mathcal{A}_{\mu}(x)+\delta_{\mu 4} a(\boldsymbol{x}) .
$$

Using equations (5), (7), (12) and (14), we can expand the source term as follows:

$$
\int^{\beta} \mathrm{d}^{4} x K_{n}\left(\Omega^{n}-\bar{\Omega}^{n}\right)
$$




$$
\begin{aligned}
= & -\int^{\beta} \mathrm{d}^{4} x \frac{\delta \Gamma}{\delta \bar{\Omega}^{n}} \frac{1}{N} \operatorname{tr}\left[\left\{\mathcal{T} \mathrm{e}^{-\mathrm{i} \int_{0}^{\beta} \mathrm{d} x_{4}^{\prime} a} \sum_{l=0}^{\infty} \frac{1}{l !}\left(-\mathrm{i} \int_{0}^{\beta} \mathrm{d} x_{4} \mathcal{A}_{4}\left(x_{4}\right)\right)^{l}\right\}^{n}-\mathrm{e}^{-\mathrm{i} n \beta a}\right] \\
= & -\int^{\beta} \mathrm{d}^{4} x \frac{\delta \Gamma}{\delta \bar{\Omega}^{n}} \frac{1}{N}\left[-\mathrm{i} n \mathrm{e}^{-\mathrm{i} n \beta a_{m}} \frac{1}{\beta} \int_{0}^{\beta} \mathrm{d} x_{4} \frac{\mathcal{A}_{4}^{m}\left(x_{4}\right)}{\sqrt{2}}\right. \\
& -\frac{n \beta}{2} \mathrm{e}^{-\mathrm{i} n \beta a_{i}} \frac{1}{\beta} \int_{0}^{\beta} \mathrm{d} x_{4} \int_{0}^{x_{4}} \mathrm{~d} x_{4}^{\prime} \mathrm{e}^{\mathrm{i}\left(x_{4}-x_{4}^{\prime}\right)\left(a_{i}-a_{j}\right)} \mathcal{A}_{4}^{(i, j)}\left(x_{4}\right) \mathcal{A}_{4(i, j)}\left(x_{4}^{\prime}\right) \\
& \left.-\frac{1}{2} \sum_{k=1}^{n-1} k \mathrm{e}^{-\mathrm{i} n \beta a_{j}-\mathrm{i} k \beta\left(a_{i}-a_{j}\right)} \int_{0}^{\beta} \mathrm{d} x_{4} \int_{0}^{\beta} \mathrm{d} x_{4}^{\prime} \mathrm{e}^{\mathrm{i}\left(x_{4}-x_{4}^{\prime}\right)\left(a_{i}-a_{j}\right)} \mathcal{A}_{4}^{(i, j)}\left(x_{4}\right) \mathcal{A}_{4(i, j)}\left(x_{4}^{\prime}\right)\right]+\mathrm{O}\left(\mathcal{A}^{3}\right) \\
= & -\int^{\beta} \mathrm{d}^{4} x \frac{\delta \Gamma}{\delta a_{n}} \tilde{\mathcal{A}}_{4}^{n}+\int^{\beta} \mathrm{d}^{4} x \frac{1}{2}\left(\frac{\delta \Gamma}{\delta a_{n}}-\frac{\delta \Gamma}{\delta a_{m}}\right) \frac{1}{1-\mathrm{e}^{-\mathrm{i} \beta\left(a_{m}-a_{n}\right)}} \\
& \times \frac{\mathrm{i}}{\beta} \int_{0}^{\beta} \mathrm{d} x_{4} \int_{0}^{x_{4}} \mathrm{~d} x_{4}^{\prime} \mathrm{e}^{\mathrm{i}\left(x_{4}-x_{4}^{\prime}\right)\left(a_{n}-a_{m}\right)} \mathcal{A}_{4}^{(m, n)}\left(x_{4}\right) \mathcal{A}_{4(m, n)}\left(x_{4}^{\prime}\right)+\mathrm{O}\left(\mathcal{A}^{3}\right)
\end{aligned}
$$

with the notation $\mathcal{A}_{4(i, j)}$ for the $(i, j)$ component of the matrix $\mathcal{A}_{4}$ divided by $\sqrt{2}$, that is, an off-diagonal $(i, j)$ component of the ladder basis. In the last line $\tilde{\mathcal{A}}_{4}^{n}$ is defined by (6) in which $\mathcal{A}$ is substituted for $A$. If we proceed to the $n$-loop calculation of the effective action $\Gamma$, we have to use the $(n-1)$-loop result for $\Gamma$ into $\delta \Gamma / \delta a$ and at the same time the neglected terms $\mathrm{O}\left(\mathcal{A}^{3}\right)$ are also necessary.

In the final expression of (15) the first term simply removes all the one-particle reducible diagrams as in the case for the elementary gauge fields written in (44). The second term corresponds to the renormalization of the Polyakov loop, which is not the renormalization of the ultraviolet divergence and is necessary for the two-loop or higher-order calculations of the effective potential [6] 8]. We emphasize that any effect of the renormalization of the Polyakov loop automatically comes out in our formalism because we began with the appropriate definition of the effective action. Of course, it is also possible to understand the second term from the point of view of [6]. The background field $a$ is renormalized to $a+\delta a$ and then the effective action can be expanded as $\Gamma[a+\delta a]=\Gamma[a]+\delta \Gamma / \delta a \cdot \delta a$, the second term of which is the same form as the second term in (15). In the present case of the effective action the situation is slightly involved. The zero-loop order of the kinetic term (tree term) may survive so that the effective action of the one-loop order can also be affected by the renormalization of the Polyakov loop with spatial variations. However, although finite corrections may be certainly generated, they can be absorbed into the arbitrariness of the renormalization of ultraviolet divergence and thus we do not have to bother with the renormalization of the Polyakov loop up to one-loop order at all. In the next section we explore this point further.

\section{PERTuRBAtive EVAluAtion of THE EFFECTIVE ACTION}

The background field method has an advantage in that the effective action preserves the manifest gauge invariance [18]. The gauge fields are decomposed into

$$
A_{\mu}^{a}=\mathcal{A}_{\mu}^{a}+A_{\mathrm{B} \mu}^{a}
$$

where $\mathcal{A}_{\mu}^{a}$ and $A_{\mathrm{B} \mu}^{a}$ denote the quantum and the background part, respectively. Introducing the covariant derivative in terms of the background fields by $D_{\mathrm{B} \mu}=\partial_{\mu}+\mathrm{i}\left[A_{\mathrm{B} \mu}\right.$, ], one can write the gauge-fixing condition as

$$
D_{\mathrm{B} \mu} \mathcal{A}_{\mu}^{a}-\omega^{a}=0 .
$$

Multiplying a Gaussian weight and integrating $\omega^{a}$ out, we obtain the Lagrangian,

$$
\begin{aligned}
\mathcal{L}= & -\frac{1}{4 g^{2}}\left[F_{\mathrm{B} \mu \nu a} F_{\mathrm{B} \mu \nu}^{a}+4 F_{\mathrm{B} \mu \nu a} D_{\mathrm{B} \mu} \mathcal{A}_{\nu}^{a}\right. \\
& -2 \mathcal{A}_{\mu a}\left\{\left(D_{\mathrm{B}}^{2}\right)^{a}{ }_{b} \delta_{\mu \nu}-\left(1-\frac{1}{\xi}\right)\left(D_{\mathrm{B} \mu} D_{\mathrm{B} \nu}\right)^{a}{ }_{b}\right\} \mathcal{A}_{\nu}^{b} \\
& \left.-4 f_{b c}^{a} D_{\mathrm{B} \mu} \mathcal{A}_{\nu a} \mathcal{A}_{\mu}^{b} \mathcal{A}_{\nu}^{c}+f_{a b}^{c} f_{d e c} \mathcal{A}_{\mu}^{a} \mathcal{A}_{\nu}^{b} \mathcal{A}_{\mu}^{d} \mathcal{A}_{\nu}^{e}\right]+\bar{c}_{a}\left\{\left(D_{\mathrm{B}}^{2}\right)_{b}^{a}+\left(D_{\mathrm{B} \mu}\right)_{c}^{a} f_{b d}{ }_{b d} \mathcal{A}_{\mu}^{d}\right\} c^{b} .
\end{aligned}
$$

From the quadratic part of this Lagrangian, we can readily integrate the quantum fields $\mathcal{A}_{\mu}^{a}$ and $c^{a}$ in the Feynman gauge $\xi=1$ to give the one-loop effective action, 


$$
\Gamma\left[A_{\mathrm{B}}\right]=\int^{\beta} \mathrm{d}^{4} x\left(-\frac{1}{4 g^{2}} F_{\mathrm{B} \mu \nu a} F_{\mathrm{B} \mu \nu}^{a}\right)-\frac{1}{2} \operatorname{tr}^{\prime} \ln \left\{\left(D_{\mathrm{B}}^{2}\right)_{a b} \delta_{\mu \nu}+2 f_{a b}^{c} F_{\mathrm{B} \mu \nu c}\right\}+\operatorname{tr} \ln \left\{D_{\mathrm{B}}^{2}\right\}
$$

where $\operatorname{tr}$ is the trace of the spatial and colour indices, while $t^{\prime}$ includes the trace over the Lorentz indices in addition. We set the background field as (14) and $a$ is decomposed into

$$
a(\boldsymbol{x})=\frac{1}{\beta}(C+\epsilon(\boldsymbol{x}))
$$

where $C$ and $\epsilon(\boldsymbol{x})$ represent the constant and the variational part of the background field, respectively. They are allowed to be traceless independently, i.e.

$$
\operatorname{tr} C=\sum_{i=1}^{N} C_{i}=0 \quad \operatorname{tr} \epsilon(\boldsymbol{x})=\sum_{i=1}^{N} \epsilon_{i}(\boldsymbol{x})=0 .
$$

We divided them by $\beta$ in order to make $C$ and $\epsilon(\boldsymbol{x})$ dimensionless. In the ladder basis [7, 8] the effective action (19) is attained most simply as the following:

$$
\begin{aligned}
\Gamma[C, \epsilon]= & \frac{V}{\beta^{3} N g^{2}} \sum_{i>j} \epsilon_{i j} \nabla^{2} \epsilon_{i j}-\frac{1}{2} \operatorname{tr}^{\prime} \ln \left[\partial_{(i, j)}^{2} \delta_{\mu \nu}+2 \mathrm{i}\left\{\left(\partial_{\mu} \delta_{\nu 4}-\delta_{\mu 4} \partial_{\nu}\right) \epsilon_{i j}\right\}\right. \\
& \left.+2 \mathrm{i} \epsilon_{i j} \delta_{\mu \nu} \partial_{4(i, j)}-\left(\epsilon_{i j}\right)^{2} \delta_{\mu \nu}\right]+\operatorname{tr} \ln \left\{\partial_{(i, j)}^{2}+2 \mathrm{i} \epsilon_{i j} \partial_{4(i, j)}-\left(\epsilon_{i j}\right)^{2}\right\}
\end{aligned}
$$

where we rescaled all the coordinate variables by $\beta$ so as to let them be dimensionless. From now on, we consider all the coordinates, momenta and fields as dimensionless multiplied appropriately by $\beta$. Here we denote the dimensionless derivatives with the background fields as

$$
\begin{array}{ll}
\partial_{\mu(i, j)}=\partial_{\mu}+\mathrm{i} \delta_{\mu 4} C_{i j} & p_{\mu(i, j)}=p_{\mu}+\delta_{\mu 4} C_{i j} \\
C_{i j}=C_{i}-C_{j} & \epsilon_{i j}=\epsilon_{i}-\epsilon_{j} .
\end{array}
$$

Our purpose is to expand the effective action (22) in powers of $\epsilon$. In terms of Feynman's diagrammatical method, the terms with $\epsilon$ and $\epsilon^{2}$ in the action (22) are interpreted as the three- and four-point interaction vertices. The quadratic derivative terms correspond to the free propagator.

The one-loop effective potential shown diagrammatically in Fig. 1 has already been argued in [5] with the result,

$$
\begin{aligned}
\Gamma^{(0)}[C] & =-\frac{V}{2 \beta^{3}} \int \frac{\mathrm{d}^{3} p}{(2 \pi)^{3}} \sum_{n} \operatorname{tr}^{\prime} \ln \left\{p_{(i, j)}^{2} \delta_{\mu \nu}\right\}+\frac{V}{\beta^{3}} \int \frac{\mathrm{d}^{3} p}{(2 \pi)^{3}} \sum_{n} \operatorname{tr} \ln \left\{p_{(i, j)}^{2}\right\} \\
& =-\frac{\pi^{2} V}{12 \beta^{3}} \sum_{i>j}\left(\frac{C_{i j}}{\pi}\right)_{\bmod 2}^{2}\left\{\left(\frac{C_{i j}}{\pi}\right)_{\bmod 2}-2\right\}^{2}
\end{aligned}
$$

where $n$ in the summation is the Matsubara frequency, which appears only through the momentum variables $p_{(i, j)}=$ $\left(2 \pi n+C_{i j}, \boldsymbol{p}\right)$.

\section{A. Up to quadratic order}

The first correction up to $\epsilon^{2}$ order is represented diagrammatically in Fig. 2. Introducing the Fourier transform of the variational fields,

$$
\epsilon_{i j}(\boldsymbol{x})=\int \frac{\mathrm{d}^{3} q}{(2 \pi)^{3}} \epsilon_{i j}(\boldsymbol{q}) \mathrm{e}^{-\mathrm{i} q \cdot x}
$$

we can easily write the tree term as

$$
\Gamma_{\text {tree }}[\epsilon]=-\frac{V}{\beta^{3} N g^{2}} \sum_{i>j} \int \frac{\mathrm{d}^{3} q}{(2 \pi)^{3}}\left|\epsilon_{i j}(\boldsymbol{q})\right|^{2} \boldsymbol{q}^{2} .
$$


The procedure to calculate the loop corrections is almost the same as that in [7] except for the absence of the condition that the momentum is sufficiently small. This condition is really the case for the $\mathrm{Z}(N)$ domain wall but in contrast with it our results should contain all orders of the derivative expansion so that it would not take the form of the ordinary kinetic term proportional to momentum squared. The renormalized contribution to the effective action is calculated as

$$
\begin{aligned}
\Gamma_{\text {loop }}^{(2)}[C, \epsilon] & =\frac{V}{\beta^{3}} \sum_{i, j} \int \frac{\mathrm{d}^{3} q}{(2 \pi)^{3}}\left|\epsilon_{i j}(\boldsymbol{q})\right|^{2} \sum_{n} \int \frac{\mathrm{d}^{3} p}{(2 \pi)^{3}}\left\{\frac{2\left(p_{4(i, j)}^{2}+q^{2}\right)}{\left(p_{(i, j)}+q\right)^{2}}-1\right\} \frac{1}{p_{(i, j)}^{2}} \\
& =-\frac{V}{\beta^{3}} \sum_{i>j} \int \frac{\mathrm{d}^{3} q}{(2 \pi)^{3}}\left|\epsilon_{i j}(\boldsymbol{q})\right|^{2}\left\{\frac{11}{48 \pi^{2}} q^{2} \ln \frac{q^{2}}{M^{2}}+\frac{1}{4 \pi} f\left(q, C_{i j}\right)\right\}
\end{aligned}
$$

where we used the 4 -vector notation $q=(0, \boldsymbol{q}) . \quad M$ is a renormalization point. The first term in brackets in (28) comes from the vacuum part and the second one comes from the matter part which vanishes as $T \rightarrow 0$.

The function $f(q, C)$ is defined by

$$
\begin{aligned}
f(q, C) & =\frac{2}{\pi} \int_{0}^{\infty} \mathrm{d} p\left\{p+q\left(1-\frac{p^{2}}{q^{2}}\right) \ln \left|\frac{2 p-q}{2 p+q}\right|\right\} N\left(p, C_{i j}\right), \\
N\left(p, C_{i j}\right) & =\frac{1}{\mathrm{e}^{p+\mathrm{i} C_{i j}}-1}+\frac{1}{\mathrm{e}^{p-\mathrm{i} C_{i j}}-1},
\end{aligned}
$$

whose behaviour is shown in Fig. 3. $N(p, C)$ is the distribution function which is reduced to the usual Bose distribution function at $C=0$ and to the negative Fermi distribution function at $C=\pi$. This exchange of the statistical character agrees with the centre-symmetric treatment proposed recently in which the charged gluon must obey the antiperiodic boundary condition [15] (of course, the origin is actually identical). If the coefficient of $N(p, C)$ is positive definite and also sufficiently large, the minimum of the effective action will shift into $C \sim \pi$, i.e. the confining vacuum. In our formalism the result of [12, 13] can be interpreted as that non-zero value of $\left|\epsilon_{i j}(\boldsymbol{q})\right|$ causes $C_{i j} \rightarrow \pi$ as the temperature is lowered. However, as we will discuss in the subsequent sections, our consequence suggests another scenario: $C_{i j}$ remains zero and only $\left|\epsilon_{i j}(\boldsymbol{q})\right|$ makes the Polyakov loop decrease. Furthermore, the behaviour in the vicinity of $C=\pi$ will prove to be so infrared singular for spatial variations that it is out of reach of the perturbative regime.

We have to explain now that the renormalization of the Polyakov loop is actually harmless to the above result, as mentioned before. The second term of (15) corresponds to the first correction of the Taylor expansion in the variation from the renormalization of the Polyakov loop, that is, $\delta \epsilon$. It is possible to calculate $\delta \epsilon$ from (15) at the lowest order of $\epsilon$ to convince ourselves that $\delta \epsilon$ is independent of $C_{i j}$ so that the correction to the effective action takes the form of (constant) $\times q^{2}\left|\epsilon_{i j}(\boldsymbol{q})\right|^{2}$ for the quadratic order of $\epsilon$. This can be absorbed by the redefinition of $M$. Consequently, we can safely ignore any effect of the renormalization of the Polyakov loop up to one-loop order.

The above result can be compacted into

$$
\Gamma^{(2)}[C, \epsilon]=-\frac{V}{\beta^{3}} \sum_{i>j} \int \frac{\mathrm{d}^{3} q}{(2 \pi)^{3}}\left|\epsilon_{i j}(\boldsymbol{q})\right|^{2}\left\{\frac{q^{2}}{N g^{2}(q, t)}+\frac{1}{4 \pi} f\left(q, C_{i j}\right)\right\}
$$

using the running coupling constant,

$$
g^{2}(q, t)=\frac{g^{2}}{1+\left(11 N g^{2} / 48 \pi^{2}\right) \ln \left(q^{2} / M^{2}\right)}=\frac{48 \pi^{2}}{11 N \ln \left(q^{2} / \beta^{2} \Lambda_{0}^{2}\right)}=\frac{24 \pi^{2}}{11 N \ln (q t)}
$$

where $t$ denotes the dimensionless temperature in units of $\Lambda_{0}$, the QCD scale parameter.

Asymptotic values of $f(q, C)$ are easily obtained as

$$
\begin{aligned}
f(q \rightarrow 0, C) & =\frac{4 \pi}{3}-4 C+\frac{2 C^{2}}{\pi} \\
f(q \rightarrow \infty, C) & =-\frac{3}{2} f(q \rightarrow 0, C)=-2 \pi+6 C-\frac{3 C^{2}}{\pi}
\end{aligned}
$$

It is obvious that (33) at $C=0$ provides the correct perturbative result of the Debye mass which stabilizes the perturbative vacuum. The behaviour of (34) that $f(q, C)$ turns to be negative as $q$ becomes larger is crucial in our argument. The perturbative vacuum $\left(C_{i j}=\epsilon_{i j}(\boldsymbol{q})=0\right)$ may become unstable supposing that the decreasing rate of $f(q, C)$ overcomes the increasing rate of the tree term (quadratic in momentum). Actually, it is the case when the coupling constant is beyond some critical value. 
Both $C_{i j}$ and $\epsilon_{i j}$ are determined by the condition that $-\Gamma[C, \epsilon]$ (the free energy in thermodynamics) should be minimized. From (31) it follows that the vacuum stability for a given $\epsilon(\boldsymbol{q})$ around the perturbative vacuum depends on the sign of

$$
h(q, t)=\frac{1}{4 \pi}\left\{\frac{q^{2}}{N \alpha(q, t)}+f(q, C=0)\right\}
$$

with $\alpha(q, t)=g^{2}(q, t) / 4 \pi$. Of course $\epsilon_{i j}$ is not independent of each other under the constraint (13). At $C=0$, however, the coefficient function $h(q, t)$ contains no colour dependence (ij dependence) so that a negative $h(q, t)$ is sufficient for the occurrence of the vacuum instability. The behaviour of $h(q, t)$ in the case of $\mathrm{SU}(3)(N=3)$ as the temperature $t$ varies is shown in Fig. 1 .

Perturbative calculations are not reliable at all for $\alpha(q, t)>1$. Looking at Fig. 1 we realize that the momentum region with $\alpha>1$ indicated by the broken curves grows when we lower the temperature and at the same time the possible unstable modes in which $h(q, t)<0$ come to spread. Therefore, whether our perturbative evaluation is reliable or not near the unstable modes depends on whether the spreading rate of the unstable modes overcomes the

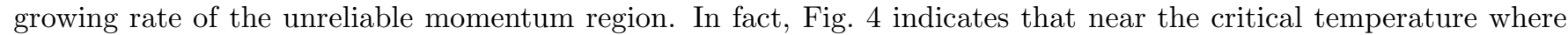
unstable modes begin appearing, the relevant modes are in the momentum region for $\alpha<1$. To be more precise, at the critical temperature $T_{\mathrm{c}}=2.77 \Lambda_{0}$, the unstable mode is $q_{\mathrm{c}}=1.33$ and the corresponding critical coupling constant is $\alpha_{\mathrm{c}}=1.31 / N$. In the case of $\mathrm{SU}(3)$, for example, $\alpha_{\mathrm{c}}=0.44$ which is surely smaller than one.

However, it is subtle to decide from the critical value of the coupling constant whether or not the perturbative calculation works well at that point. Nevertheless it is most likely that this instability actually occurs because we can comprehend it in an intuitive manner as follows. Under the effect of the temperature, real (on-shell) particles are excited thermally. The average distance between the thermal particles is of order of $1 / T$ and their distribution is incoherent. Owing to this incoherency the vacuum tends to be stable against spatial variations with small momentum or equivalently with larger-scale variations compared with the average distance between thermal particles. For variations with momentum larger than $\sim T$, on the other hand, the vacuum tends toward instability as the overlap of the vacuum configuration with the distribution of thermal particles increases. If the strength of the interaction, that is to say, the tendency to overlap the configuration is large enough, the vacuum may become unstable.

Once unstable modes emerge, the variational fields $\epsilon_{i j}$ go on increasing to minimize the free energy unless the higher terms are taken into account. In order to investigate the property of the instability more closely we need higher-order terms of $\epsilon_{i j}$. This is the subject dealt with in the next subsection.

\section{B. Up to quartic order}

Corresponding diagrams to be evaluated are displayed in Fig. 5. The result obtained after tedious calculations has singularities at $C=0$, whereas the quartic contributions are ultraviolet and infrared finite as long as $C \neq 0$. The reason for the presence of singularities is quite obvious: since we expanded the effective action in powers of $\epsilon /(C$ or $T)$, our expansion is not valid at $C=0$ for the temporal zero-mode for which $T$ is absent. The centrifugal barrier at $C=0$ observed in 12,13 seems to arise from these spurious singularities. Therefore, we must manage infrared divergence by resumming zero modes, as in the case of ring resummation for infrared divergence. We remark upon this problem in the next subsection.

In this subsection we evaluate the variational term of quartic order from which all the temporal zero-mode terms are subtracted to be resummed afterwards. Once we get rid of the zero mode it can be expected that the result will be almost independent of $q$ in the interested region $q \sim 1.3$, because the expansion in terms of $q$ becomes a power series of

$$
\frac{2 \boldsymbol{p} \cdot \boldsymbol{q}}{(2 \pi n)^{2}+\boldsymbol{p}^{2}} \quad \text { or } \quad \frac{\boldsymbol{q}^{2}}{(2 \pi n)^{2}+\boldsymbol{p}^{2}}
$$

where $\boldsymbol{p}$ is the momentum to be integrated and $n$ is the Matsubara frequency. Although in the case of $n=0$ they are singular near $\boldsymbol{p} \sim 0$, we can regard them as small enough as far as $n \neq 0$ and $q \sim 1.3$. Of course, it is possible to compute the quartic correction directly from the corresponding diagrams. We confirmed numerically that the result, which is too intricate an expression to write down here, hardly depends on $q$ around the unstable region.

Now that the result proved to be almost $q$-independent we can evaluate the quartic correction at the limit of $q \rightarrow 0$.

$$
\Gamma^{(4)}[C, \epsilon] \rightarrow-\frac{V}{\beta^{3}} \sum_{i j} \int \frac{\mathrm{d}^{3} q_{1}}{(2 \pi)^{3}} \frac{\mathrm{d}^{3} q_{2}}{(2 \pi)^{3}} \frac{\mathrm{d}^{3} q_{3}}{(2 \pi)^{3}} \epsilon_{i j}\left(\boldsymbol{q}_{1}\right) \epsilon_{i j}\left(\boldsymbol{q}_{2}\right) \epsilon_{i j}\left(\boldsymbol{q}_{3}-\boldsymbol{q}_{1}\right) \epsilon_{i j}\left(-\boldsymbol{q}_{2}-\boldsymbol{q}_{3}\right)
$$




$$
\begin{aligned}
& \times \int \frac{\mathrm{d}^{3} p}{(2 \pi)^{3}} \sum_{n \neq 0}\left[\frac{4 p_{4(i, j)}^{4}}{p_{(i, j)}^{8}}+\frac{4 p_{4(i, j)}^{2}}{p_{(i, j)}^{6}}+\frac{1}{2 p_{(i, j)}^{4}}\right] \\
= & -\frac{V}{\beta^{3}} \sum_{i>j} \int \frac{\mathrm{d}^{3} q_{1}}{\left(2 \pi^{3}\right)} \cdots \int \frac{\mathrm{d}^{3} p}{(2 \pi)^{3}} \\
& \times \int_{-\infty+\mathrm{i} \eta}^{\infty+\mathrm{i} \eta} \frac{\mathrm{d} p_{4}}{(2 \pi)}\left[\frac{8 p_{4}^{4}}{p^{8}}+\frac{8 p_{4}^{2}}{p^{6}}+\frac{1}{p^{4}}\right]\left(1+\frac{1}{\mathrm{e}^{-\mathrm{i} p_{4}+\mathrm{i} C_{i j}-1}}+\frac{1}{\mathrm{e}^{-\mathrm{i} p_{4}-\mathrm{i} C_{i j}-1}}\right) \\
= & -\frac{V}{\beta^{3}} \sum_{i>j} \int \frac{\mathrm{d}^{3} q_{1}}{(2 \pi)^{3}} \cdots \int \frac{\mathrm{d}^{3} p}{(2 \pi)^{3}} \frac{1}{12} \frac{\partial^{3}}{\partial p^{3}}\left(1+\frac{1}{\mathrm{e}^{p+\mathrm{i} C_{i j}-1}}+\frac{1}{\mathrm{e}^{p-\mathrm{i} C_{i j}-1}}\right) \\
= & -\frac{V}{\beta^{3}} \sum_{i>j} \int \frac{\mathrm{d}^{3} q_{1}}{(2 \pi)^{3}} \frac{\mathrm{d}^{3} q_{2}}{(2 \pi)^{3}} \frac{\mathrm{d}^{3} q_{3}}{(2 \pi)^{3}} \epsilon_{i j}\left(\boldsymbol{q}_{1}\right) \epsilon_{i j}\left(\boldsymbol{q}_{2}\right) \epsilon_{i j}\left(\boldsymbol{q}_{3}-\boldsymbol{q}_{1}\right) \epsilon_{i j}\left(-\boldsymbol{q}_{2}-\boldsymbol{q}_{3}\right) \frac{1}{12 \pi^{2}} .
\end{aligned}
$$

The first, second and third term in the square bracket corresponds to Fig. 司 $(a)-(c)$, respectively. The result (37) is nothing but the coefficient of $C^{4}$ in the Weiss potential (25). Since the Weiss potential is a quartic polynomial, we can realize without any calculation that terms of order higher than quartic are never relevant under the soft momentum limit if subtracted by their zero mode.

\section{Zero-mode resummation}

One may wonder why we did not take account of the cubic terms of $\epsilon$ in spite of the fact that the Weiss potential (25) has the cubic term $C^{3}$. If we compute them perturbatively as in the same manner for quadratic or quartic order, however, they are always zero: the integrand for the cubic order is proportional to $p_{4(i, j)}$ so that the summation over the Matsubara frequency and the colour indices leads to zero. Then where does the cubic term in the potential (25) come from? The answer is the zero-mode resummation, which is quite similar to the $e^{3}$-correction of the QED partition function [19].

Here we will perform the zero-mode resummation only for the most dangerous terms that are divergent in the infrared limit. The total momentum insertion from the variational fields $\epsilon$ is necessarily zero for one blob (self-energy part) so that each propagator from one blob to the other possesses the same momentum to bring about the strongest infrared singularities, as is represented in Fig. 6.

Taking away all the terms with $n \neq 0$ and subtracting quadratic terms (infrared finite terms), we reduce the effective action (22) into

$$
\begin{aligned}
-\frac{1}{4} \operatorname{tr}^{\prime} \ln \left[\delta_{\mu \nu}-\frac{2 \epsilon_{i j}^{2}}{\partial_{(i, j)}^{2}} \delta_{\mu \nu}-\frac{4 C_{i j}^{2} \epsilon_{i j}}{\partial_{(i, j)}^{2}} \frac{\epsilon_{i j}}{\partial_{(i, j)}^{2}} \delta_{\mu \nu}+\frac{4\left\{\left(\partial_{\mu} \delta_{\lambda 4}-\delta_{\mu 4} \partial_{\lambda}\right) \epsilon_{i j}\right\}}{\partial_{(i, j)}^{2}} \frac{\left\{\left(\partial_{\lambda} \delta_{\nu 4}-\delta_{\lambda 4} \partial_{\nu}\right) \epsilon_{i j}\right\}}{\partial_{(i, j)}^{2}}\right. \\
\left.+\frac{4 \mathrm{i} C_{i j} \epsilon_{i j}}{\partial_{(i, j)}^{2}} \frac{\left\{\left(\partial_{\mu} \delta_{\nu 4}-\delta_{\mu 4} \partial_{\nu}\right) \epsilon_{i j}\right\}}{\partial_{(i, j)}^{2}}+\frac{4 \mathrm{i} C_{i j}\left\{\left(\partial_{\mu} \delta_{\nu 4}-\delta_{\mu 4} \partial_{\nu}\right) \epsilon_{i j}\right\}}{\partial_{(i, j)}^{2}} \frac{\epsilon_{i j}}{\partial_{(i, j)}^{2}}+\frac{\epsilon_{i j}^{2}}{\partial_{(i, j)}^{2}} \frac{\epsilon_{i j}^{2}}{\partial_{(i, j)}^{2}} \delta_{\mu \nu}\right] \\
+\frac{1}{2} \operatorname{tr} \ln \left[1-\frac{2 \epsilon_{i j}^{2}}{\partial_{(i, j)}^{2}}-\frac{4 C_{i j}^{2} \epsilon_{i j}}{\partial_{(i, j)}^{2}} \frac{\epsilon_{i j}}{\partial_{(i, j)}^{2}}+\frac{\epsilon_{i j}^{2}}{\partial_{(i, j)}^{2}} \frac{\epsilon_{i j}^{2}}{\partial_{(i, j)}^{2}}\right]-\left(\epsilon_{i j}^{2} \text {-terms }\right)
\end{aligned}
$$

where any term of $\epsilon^{3}$ is absent because all the diagrams possessing such vertices are to be resummed individually to contribute either to the order of $\epsilon^{5}$ or to less singular terms. In order to evaluate (38) we perform calculations in the momentum space. $\epsilon(\boldsymbol{x})$ is sum of all the modes $\epsilon(\boldsymbol{q})$, among which we leave only the most singular combination, as shown in Fig. 6.

To take the trace for the Lorentz indices, it is useful to decompose them into three projective components, namely $P_{\mathrm{T} \mu \nu}=\delta_{\mu \nu}-q_{\mu} q_{\nu} / q^{2}-\delta_{4 \mu} \delta_{4 \nu}, P_{\mathrm{L} \mu \nu}=q_{\mu} q_{\nu} / q^{2}$ and $u_{\mu \nu}=\delta_{4 \mu} \delta_{4 \nu}$. Then (38) can be rewritten as

$$
\begin{aligned}
& -\frac{V}{4 \beta^{3}} \sum_{i, j} \int \frac{\mathrm{d}^{3} p}{(2 \pi)^{3}} \sum_{\mu=\nu} \ln \left[\delta_{\mu \nu}+\left\{\int \frac{\mathrm{d}^{3} q}{(2 \pi)^{3}}\left|\epsilon_{i j}(\boldsymbol{q})\right|^{2}\left(\frac{2}{p_{(i j)}^{2}}-\frac{4 C_{i j}^{2}}{p_{(i, j)}^{2}\left(p_{(i, j)}+q\right)^{2}}\right)\right.\right. \\
& \left.+\frac{1}{p_{(i, j)}^{4}}\left(\int \frac{\mathrm{d}^{3} q}{(2 \pi)^{3}}\left|\epsilon_{i j}(\boldsymbol{q})\right|^{2}\right)^{2}\right\} P_{\mathrm{T} \mu \nu}+\left\{\int \frac{\mathrm{d}^{3} q}{(2 \pi)^{3}}\left|\epsilon_{i j}(\boldsymbol{q})\right|^{2}\left(\frac{2}{p_{(i, j)}^{2}}-\frac{4\left(C_{i j}^{2}+q^{2}\right)}{p_{(i, j)}^{2}\left(p_{(i, j)}+q\right)^{2}}\right)\right.
\end{aligned}
$$




$$
\begin{aligned}
& \left.\left.+\frac{1}{p_{(i, j)}^{4}}\left(\int \frac{\mathrm{d}^{3} q}{(2 \pi)^{3}}\left|\epsilon_{i j}(\boldsymbol{q})\right|^{2}\right)^{2}\right\}\left(P_{\mathrm{L} \mu \nu}+u_{\mu \nu}\right)\right] \\
+ & \frac{V}{2 \beta^{3}} \sum_{i, j} \int \frac{\mathrm{d}^{3} p}{(2 \pi)^{3}} \sum_{\mu=\nu} \ln \left[1+\int \frac{\mathrm{d}^{3} q}{(2 \pi)^{3}}\left|\epsilon_{i j}(\boldsymbol{q})\right|^{2}\left(\frac{2}{p_{(i, j)}^{2}}-\frac{4 C_{i j}^{2}}{p_{(i, j)}^{2}\left(p_{(i, j)}+q\right)^{2}}\right)\right. \\
& \left.+\frac{1}{p_{(i, j)}^{4}}\left(\int \frac{\mathrm{d}^{3} q}{(2 \pi)^{3}}\left|\epsilon_{i j}(\boldsymbol{q})\right|^{2}\right)^{2}\right]-\left(\epsilon_{i j}^{2} \text {-terms }\right) \\
=-\frac{V}{2 \beta^{3}} & \sum_{i, j} \int \frac{\mathrm{d}^{3} p}{(2 \pi)^{3}}\left[\operatorname { l n } \left\{1+\int \frac{\mathrm{d}^{3} q}{(2 \pi)^{3}}\left|\epsilon_{i j}(\boldsymbol{q})\right|^{2}\left(\frac{2}{p_{(i, j)}^{2}}-\frac{4\left(C_{i j}^{2}+q^{2}\right)}{p_{(i, j)}^{2}\left(p_{(i, j)}+q\right)^{2}}\right)\right.\right. \\
& \left.\left.+\frac{1}{p_{(i, j)}^{4}}\left(\int \frac{\mathrm{d}^{3} q}{(2 \pi)^{3}}\left|\epsilon_{i j}(\boldsymbol{q})\right|^{2}\right)^{2}\right\}-\int \frac{\mathrm{d}^{3} q}{(2 \pi)^{3}}\left|\epsilon_{i j}(\boldsymbol{q})\right|^{2}\left(\frac{2}{p_{(i, j)}^{2}}-\frac{4\left(C_{i j}^{2}+q^{2}\right)}{p_{(i, j)}^{2}\left(p_{(i, j)}+q\right)^{2}}\right)\right] .
\end{aligned}
$$

We restrict ourselves to considering the case of $C_{i j}=0$ because no infrared singularity emerges otherwise. For an isolated mode $\boldsymbol{q}$, the zero-mode action (39) is simplified as

$$
-\frac{V}{2 \beta^{3}} \sum_{i, j} \int \frac{\mathrm{d}^{3} p}{(2 \pi)^{3}}\left[\ln \left\{1+\left|\epsilon_{i j}\right|^{2}\left(\frac{2}{p^{2}}-\frac{4 q^{2}}{p^{2}(p+q)^{2}}\right)+\frac{\left|\epsilon_{i j}\right|^{4}}{p^{4}}\right\}-\left|\epsilon_{i j}\right|^{2}\left(\frac{2}{p^{2}}-\frac{4 q^{2}}{p^{2}(p+q)^{2}}\right)\right] .
$$

It is easy to reproduce the cubic term in the Weiss potential (25) with $\boldsymbol{q}=0$. In this limiting case (40) is reduced to

$$
\begin{aligned}
& =-\frac{V}{\beta^{3}} \sum_{i, j} \frac{1}{2 \pi^{2}} \int_{0}^{\infty} \mathrm{d} p\left\{p^{2} \ln \left(1+\frac{\left|\epsilon_{i j}\right|^{2}}{p^{2}}\right)-\left|\epsilon_{i j}\right|^{2}\right\} \\
& =\frac{V}{\beta^{3}} \sum_{i, j} \frac{1}{3 \pi^{2}} \int_{0}^{\infty} \mathrm{d} p \frac{\left|\epsilon_{i j}\right|^{4}}{p^{2}+\left|\epsilon_{i j}\right|^{2}}=\frac{V}{\beta^{3}} \sum_{i>j} \frac{\left|\epsilon_{i j}\right|^{3}}{3 \pi} .
\end{aligned}
$$

This is certainly identical to the cubic term in the Weiss potential.

If the relevant mode $\boldsymbol{q}$ is non-zero then the situation changes drastically. For sufficiently small values of $\left|\epsilon_{i j}\right| \ll q$ the main contribution of the integration over $p$ stems from singularities near $p \sim 0 \sim\left|\epsilon_{i j}\right|$. Then a similar calculation as above leads to

$$
\begin{aligned}
& \simeq-\frac{V}{\beta^{3}} \sum_{i, j} \frac{1}{2 \pi^{2}} \int_{0}^{\infty} \mathrm{d} p\left\{p^{2} \ln \left(1-\frac{\left|\epsilon_{i j}\right|^{2}}{p^{2}}\right)+\left|\epsilon_{i j}\right|^{2}\right\} \\
& =\frac{V}{\beta^{3}} \sum_{i, j} \frac{1}{3 \pi^{2}} \int_{0}^{\infty} \mathrm{d} p \frac{\left|\epsilon_{i j}\right|^{4}}{p^{2}-\left|\epsilon_{i j}\right|^{2}}=0 .
\end{aligned}
$$

The integration in the last line is defined in the sense of Cauchy's principal value. The result (42) is, in fact, what is expected: the $\epsilon^{3}$-term is generated by the most severe infrared singularities. Once $q$ takes a non-vanishing value, infrared singularities within the blobs (not the propagators, see Fig. 6) are loosened.

The result obtained up to now is valid only for $\left|\epsilon_{i j}\right| \ll q$. In the next section, we want to deal with not so small $\epsilon$ around $q_{\mathrm{c}}$, beyond which the perturbative evaluation will lose its validity as shown in Fig. ㄱ. If the contribution from the zero-mode resummation comes to be opposite to that from the quartic term for some marginal value of $\epsilon$, the zero-mode resummation may cause qualitative changes: the absolute minimum of our effective action may jump with a finite gap. In fact, however, the zero-mode resummation rapidly grows as $q$ increases from zero so that it becomes positive contribution (the same sign as the quartic term) in the momentum region of interest. No qualitative picture can be affected by the zero-mode resummation. Thus for the simplicity we will entirely ignore this contribution in the next section. Although this neglection seems too rough, closer investigation of the zero-mode resummation reveals that it is not so crude. Numerical cancellation between the resummed contribution performed here and the next-to-leading contribution occurs.

The expansion singularities of $\epsilon / C$ resummed in this subsection have much to do with the famous problem of Linde's infrared catastrophe [20]. Since Fig. 2 is the same as the diagram for the gluon vacuum polarization, the two- or higher-loop calculation should break down and non-perturbative effects are no longer under control perturbatively. The infrared singularities would be regulated and perturbative expansion would work well only provided that the 
constant background field $C$ is of order of $g^{0}$ or at least $g^{1}$ as is discussed in [8]. It is the reason why the hard thermal loops (HTLs) are not necessary for the evaluation of the effective potential. The naive order counting by the coupling constant will work due to the presence of the background. Since the stationary value of the background is zero, in fact, there seems to be a logical contradiction in this argument. In our formalism the variational background field $\epsilon$ instead of $C$ takes a non-zero value, as seen below. Linde's problem is regulated here into the expansion singularities of $\epsilon / C$ that can be resummed in principle. Therefore, we can consider that our perturbative expansion of the order of

$g$ should make sense (this does not say nothing about the reliability of the perturbative evaluation) and expect that a non-perturbative contribution will be suppressed once instability occurs.

\section{NUMERICAL ANALYSIS}

The free energy density obtained above is

$$
\begin{aligned}
U[C, \epsilon]= & -\frac{\beta^{3}}{V}\left(\Gamma^{(0)}[C]+\Gamma^{(2)}[C, \epsilon]+\Gamma^{(4)}[\epsilon]\right) \\
= & \sum_{i>j}\left[\frac{\pi^{2}}{12}\left(\frac{C_{i j}}{\pi}\right)_{\bmod 2}^{2}\left\{\left(\frac{C_{i j}}{\pi}\right)_{\bmod 2}-2\right\}^{2}+\int \frac{\mathrm{d}^{3} q}{(2 \pi)^{2}}\left|\epsilon_{i j}(\boldsymbol{q})\right|^{2} \frac{1}{4 \pi}\left\{\frac{q^{2}}{N \alpha(q, t)}+f\left(q, C_{i j}\right)\right\}\right. \\
& \left.+\frac{1}{12 \pi^{2}} \int \frac{\mathrm{d}^{3} q_{1}}{(2 \pi)^{3}} \frac{\mathrm{d}^{3} q_{2}}{(2 \pi)^{3}} \frac{\mathrm{d}^{3} q_{3}}{(2 \pi)^{3}} \epsilon_{i j}\left(\boldsymbol{q}_{1}\right) \epsilon_{i j}\left(\boldsymbol{q}_{2}\right) \epsilon_{i j}\left(\boldsymbol{q}_{3}-\boldsymbol{q}_{1}\right) \epsilon_{i j}\left(-\boldsymbol{q}_{2}-\boldsymbol{q}_{3}\right)\right]
\end{aligned}
$$

We can acquire the equations of motion from the stationary conditions for the free energy, i.e. $\delta U / \delta C_{i j}=\delta U / \delta \epsilon_{i j}(\boldsymbol{q})=$ 0 and, in principle, solve them self-consistently. It is, however, quite involved because of the mode couplings induced by the nonlinear term $\epsilon^{4}$. To simplify the analysis and investigate the situation in a comprehensible manner we assume that $\epsilon^{2}(\boldsymbol{x})$ is such a function that the variance from the spatial mean value is small enough to be negligible, that is,

$$
\frac{\beta^{3}}{V} \int \mathrm{d}^{3} x\left\{\epsilon_{i j}^{2}(\boldsymbol{x})-\frac{1}{V} \int \mathrm{d}^{3} x \epsilon_{i j}^{2}(\boldsymbol{x})\right\}^{2} \simeq 0 .
$$

Then the nonlinear term takes the form,

$$
\frac{1}{12 \pi^{2}}\left(\int \frac{\mathrm{d}^{3} q}{(2 \pi)^{3}}\left|\epsilon_{i j}(\boldsymbol{q})\right|^{2}\right)^{2}
$$

This assumption is correct provided that $\epsilon(\boldsymbol{x})$ is a step-like function or has no sharp peak.

For simplicity we shall initially discuss the $\mathrm{SU}(2)$ case where $\epsilon_{2}=-\epsilon_{1}=\epsilon / 2$ and $C_{2}=-C_{1}=C / 2$. From the equations of motion one can easily obtain

$$
\int \frac{\mathrm{d}^{3} q}{(2 \pi)^{3}}|\epsilon(\boldsymbol{q})|^{2}=-6 \pi^{2} \tilde{h}(C, t)
$$

where $\tilde{h}(C, t)$ is defined as

$$
\tilde{h}(C, t)=\frac{1}{4 \pi}\left\{\frac{q_{0}^{2}(C, t)}{N \alpha\left(q_{0}(C, t), t\right)}+f\left(q_{0}(C, t), C\right)\right\},
$$

with $q_{0}(C, t)$ determined in such a way that $q^{2} / N \alpha(q, t)+f(q, C)$ should take its minimum value to minimize $U[C, \epsilon]$. Then the free energy density is written only in terms of $C$ as

$$
U[C]=U^{(0)}[C]-3 \pi^{2}\{\tilde{h}(C, t)\}^{2},
$$

where $U^{(0)}[C]$ is the Weiss potential (density),

$$
U^{(0)}[C]=\frac{\pi^{2}}{12}\left(\frac{C}{\pi}\right)_{\bmod 2}^{2}\left\{\left(\frac{C}{\pi}\right)_{\bmod 2}-2\right\}^{2} .
$$

The order parameter (1) takes the following form under the approximation (44) and using the stationary value (46), 


$$
\Omega=\exp \left(\frac{3 \pi^{2}}{4} \tilde{h}(C, t)\right) \cos \frac{C}{2} .
$$

Minimizing (48) leads to $C=0$ so that $\Omega$ varies only through the change of $\epsilon$ determined by (46) at $C=0$. The numerical behaviour of the order parameter as a function of $t$ is displayed in Fig. 7 .

It is straightforward to proceed to the case of $\mathrm{SU}(3)$ where the independent variables are $\epsilon_{1}, \epsilon_{2}, C_{1}$ and $C_{2}$. The traceless constraint (13) gives $\epsilon_{3}=-\epsilon_{1}-\epsilon_{2}$ and $C_{3}=-C_{1}-C_{2}$. Numerical investigation tells us that the minimum lies either at $\epsilon_{1}=\epsilon_{2}$ or at $\epsilon_{1}=-2 \epsilon_{2}\left(\epsilon_{2}=-2 \epsilon_{1}\right)$. Whichever of them we may choose, they are actually equivalent. We take the former case, namely, $\epsilon_{1}=\epsilon_{2}=\epsilon$. The stationary value in this case corresponding to (46) is

$$
\int \frac{\mathrm{d}^{3} q}{(2 \pi)^{3}}|\epsilon(\boldsymbol{q})|^{2}=-\frac{\pi^{2}}{3}\left\{\tilde{h}\left(2 C_{1}+C_{2}, t\right)+\tilde{h}\left(C_{1}+2 C_{2}, t\right)\right\}
$$

and the free energy density corresponding to (48) takes the form of

$$
\begin{gathered}
U[C]=U^{(0)}\left[C_{2}-C_{1}\right]+U^{(0)}\left[2 C_{1}+C_{2}\right]+U^{(0)}\left[C_{1}+2 C_{2}\right] \\
-\frac{3 \pi^{2}}{2}\left\{\tilde{h}\left(2 C_{1}+C_{2}, t\right)+\tilde{h}\left(C_{1}+2 C_{2}, t\right)\right\}^{2}
\end{gathered}
$$

whose minimum turns out to be at $C_{1}=C_{2}=0$. The behaviour of the order parameter shown in Fig. 7 is almost the same as in the $\mathrm{SU}(2)$ case.

It should be noted that we have discovered the possibility of instability but we cannot reach the phase transition. The phase transition is defined by the critical point above which spontaneous magnetization (order) appears. Any critical characteristics, such as the transition temperature, critical exponents and so on, are all defined at the very critical point. Thus all we can say is that Fig. 目 suggests some continuous instability. If we want to investigate the critical point, we must start from the confining vacuum as the strong-coupling limit on the lattice, for example, or some non-perturbative formalism [16].

As mentioned above, our result of the free energy density (43) cannot describe any discontinuous transition which is believed to be the case for $\mathrm{SU}(3)$. The reason for this is obvious: equation (43) does not possess any terms of $\epsilon^{3}$. Since the centre group Z(3) allows terms of $\Omega^{3}$ only in the case of $\mathrm{SU}(3), \epsilon^{3}$ terms are expected to be produced from some origins peculiar to $\mathrm{SU}(3)$.

Another possibility to lead to the discontinuity is the instability near $C \sim \pi$. Even if (43) has no odd terms of $\epsilon$ it is a complicated nonlinear function in terms of $C$ so that the discontinuous transition in terms of $C$ cannot be ruled out. Actually, Fig. 3 shows that $f(q, C=\pi)$ becomes negative in the soft momentum region, which may cause $h(q, t)$ to be negative. Although this instability seems to be similar to the case of $C \sim 0$, the relevant modes are entirely different. On one hand, in the case of $C \sim 0$, the unstable modes are around $q \sim 1.3$ that is comparable to the temperature. In the case of $C \sim \pi$, on the other hand, the instability is enhanced as the momentum goes to the soft limit $q \rightarrow 0$, where the running coupling constant at the one-loop order does not make sense. Our result that (52) has the absolute minimum at $C_{1}=C_{2}=0$ would be valid provided that the minimum value of $\tilde{h}(C=\pi, t)$ were at most $f(q \rightarrow 0, C=\pi) / 4 \pi=-1 / 6$ (we have used this assumption implicitly in searching for the minimum of (48) and (52)). However, the behaviour of $\tilde{h}(C, t)$ obtained here is only up to one-loop order and thus we have no idea what really happens near $C \sim \pi$. All we can state is that $C \sim \pi$ is certainly unstable and that the relevant momentum is so infrared that any perturbative calculations would not do well. It is therefore probable that the position of the absolute minimum jumps from $C=0$ to $\pi$ in the $\mathrm{SU}(3)$ case if the free energy density is attained in a non-perturbative way, which is beyond the scope of this paper.

The situation is clear in Fig. 8 which shows the behaviour of (48). The instability discovered here is for the modes near $C=0$ at lower temperature. Around $C \simeq \pi$ the relevant modes are so infrared that non-perturbative effects turn out to be of importance, even if the temperature is sufficiently high. It might seem curious that in Fig. 8 the potential value at $t=3.0$ is lower than that at $t=1.6$ near $C=\pi$; this is because we have searched for the most unstable mode $q_{0}$ within a range larger than $1 / t$ in order to avoid spurious negative values of the running coupling constant. When the temperature becomes larger, the lower bound of the momentum range to be searched decreases and thus the potential value also decreases.

\section{SUMMARY AND CONCLUSION}

We investigate the effective action of the Polyakov loop defined in a field-theoretic manner. The gauge invariance of the effective action can be proven by the same strategy as deriving the gauge dependence identities. Owing to the 
appropriate definition of the effective action any effect of the renormalization of the Polyakov loop that is necessary for higher-order calculations is automatically generated through non-local interaction vertices. In order to evaluate the effective action we choose the background field gauge where the background gauge symmetry remains manifestly. In this gauge the most essential properties to attain reliable results, that is, the gauge invariance and the unambiguous renormalization, are satisfied simultaneously.

In the actual calculation we expand the effective action in powers of the variational amplitudes $\epsilon(\boldsymbol{x})$ instead of the derivatives. The quadratic term of the variational field shows the possibility of the vacuum instability for marginal modes around $C=0$ or for the constant background $C \sim \pi$. The reason why the vacuum may become unstable is comprehensible from our resultant expression: as for the former case $(C=0)$, the variational background is usually absent because such a configuration costs more kinetic energy. In the thermal circumstance, however, on-shell particles are excited to obey the thermal distribution function, in addition to the quantum excitation. If the mode of the variational background becomes comparable with the inter-distance of the thermal particles, i.e. $\sim 1 / T$ and if the interaction is sufficiently strong, then the vacuum can decay into non-vanishing variational background. As for the latter case $(C \sim \pi)$ the statistical character of the transverse gluon changes into that of the Fermi particle, that is to say, the transverse gluon obeys the antiperiodic boundary condition. Moreover the distribution number of particles turns to be negative. The serious problem we encounter is the magnitude of the coupling constant. It depends on the smallness of coupling constant whether our effective action at one-loop order is reliable or not. In the SU(3) case the critical coupling constant is $\alpha_{\mathrm{c}}=0.44$. Particularly at finite temperature this value is too large to regard it as small. It is well known that the higher-order corrections to the free energy up to $g^{5}$ order (this is the upper limit of Linde's problem) is so oscillating that the perturbative series does not seem to converge at all. Nevertheless we believe that the physical picture may be reflected qualitatively in the one-loop effective action. It is an interesting and essential point that the kinetic term of the effective action even at one-loop order can bring about instability at the perturbative vacuum, which is out of reach of the conventional effective potential. The effective action at one-loop order already has a non-trivial physical content.

Once we pursue the policy of studying the physics contained in the one-loop effective action or believe in the perturbative evaluation despite the large coupling value, we can proceed to expand the effective action to quartic order. A brief discussion reveals that the quartic term is independent of modes provided that the temporal zero mode is subtracted. Looking over the Weiss potential we can see that any terms higher than quartic order come from the temporal zero mode. The zero-mode resummation is performed, where we make clear the origin of the cubic term in the Weiss potential.

The effective action density determines the stationary vacuum for the variational background $\epsilon(\boldsymbol{x})$ and the constant background $C$. The minimum lies at $C=0$ so that the Polyakov loop decreases only by non-vanishing $\epsilon$ provided that the instability near $C=\pi$ is harmless. Since we know that in the $\mathrm{SU}(2)$ case the transition is continuous (second order), the instability found in this paper is likely to be relevant to the real physics. In the SU(3) case, on the other hand, the discontinuous transition implies the possibility that the infrared instability around $C=\pi$ comes to play an essential role.

It is necessary, of course, that the qualitative argument discussed here is justified by some reliable method other than perturbative evaluations. Some methods, such as the thermal renormalization group and infrared effective theory successfully predict the non-perturbative contribution for the infrared region. The application of these methods to estimate the effective action is quite difficult due to the presence of the non-local intricate vertices corresponding to the renormalization of the Polyakov loop. That is the future work to resolve.

\section{acknowledgments}

One of the authors (KF) is supported by Research Fellowships of the Japan Society for the Promotion of Science for Young Scientists.

[1] A. M. Polyakov, Phys. Lett. 72B (1978) 477.

[2] B. Svetitsky and L. G. Yaffe, Nucl. Phys. B210[FS6] (1982) 423.

[3] B. Svetitsky, Phys. Rep. 132 (1986) 1.

[4] D. J. Gross, R. D. Pisarski and L. G. Yaffe, Rev. Mod. Phys. 53 (1981) 43.

[5] N. Weiss, Phys. Rev. D24 (1981) 475, D25 (1982) 2667. 
[6] V. M. Belyaev, Phys. Lett. 254B (1991) 153.

[7] T. Bhattacharya, A. Gocksch, C. K. Altes and R. D. Pisarski, Nucl. Phys. B383 (1992) 497.

[8] C. K. Altes, Nucl. Phys. B420 (1994) 637.

[9] J. Polónyi and K. Szlachányi, Phys. Lett. 110B (1982) 395.

[10] M. Gross, J. Bartholomew and D. Hochberg, preprint EFI 83-35-CHICAGO (1983) (unpublished).

[11] A. Gocksch and R. D. Pisarski, Nucl. Phys. B402 (1993) 657.

[12] J. Boháčik, Phys. Rev. D42 (1990) 3554.

[13] M. Engelhardt and H. Reinhardt, Phys. Lett. B430 (1998) 161.

[14] O. A. Borisenko and J. Boháčik, Phys. Rev. D56 (1997) 5086.

[15] F. Lenz and M. Thies, Ann. Phys. 269 (1998) 26.

[16] K. Fukushima, under completion.

[17] R. Kobes, G. Kunstatter and A. Rebhan, Nucl. Phys. B355 (1991) 1.

[18] L. F. Abbott, Nucl. Phys. B185 (1981) 189.

[19] J. Kupsta, Finite-Temperature Field Theory (Cambridge University Press, Cambridge, England, 1989), Chap. 5.

[20] A. D. Linde, Phys. Lett. B96 (1980) 289.

FIG. 1. Diagrams for the one-loop effective potential. The curly curves and the broken curves represent the gauge fields and the ghost fields, respectively.

FIG. 2. Diagrams up to quadratic order. The doubled curly curves denote the variational fields.

FIG. 3. The behaviour of the function $f(q, C)$ with various values of $C$.

FIG. 4. The behaviour of the function $h(q, t)$ for various temperature $t$. Broken curves represent the momentum region where $\alpha>1$.

FIG. 5. The variational corrections of quartic order.

FIG. 6. The ring diagram : each pair of doubled curly curves has the opposite momentum so that every single curly curve possesses the same momentum (inserted momentum is always zero).

FIG. 7. The behaviour of the order parameter as a function of the temperature $t$ for $\mathrm{SU}(2)$ and $\mathrm{SU}(3)$.

FIG. 8. The free energy density only in terms of $C$ : The broken curves are in the unreliable region $\alpha>1$. 
Figure 1

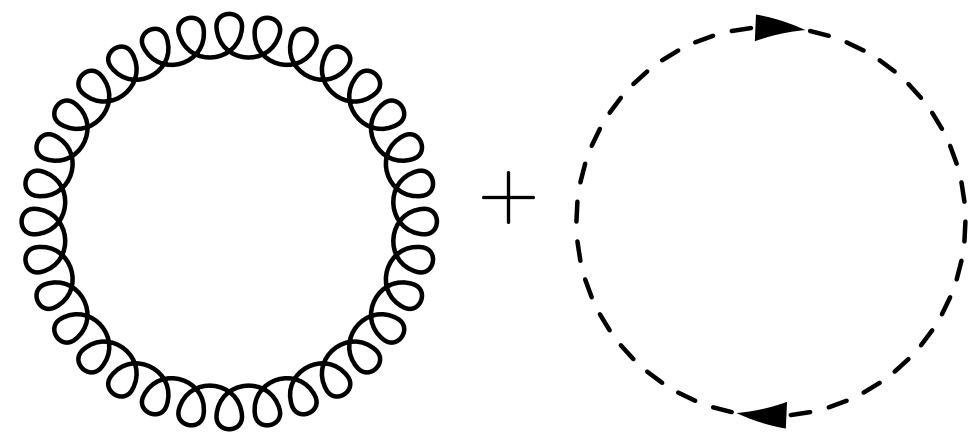

Figure 2
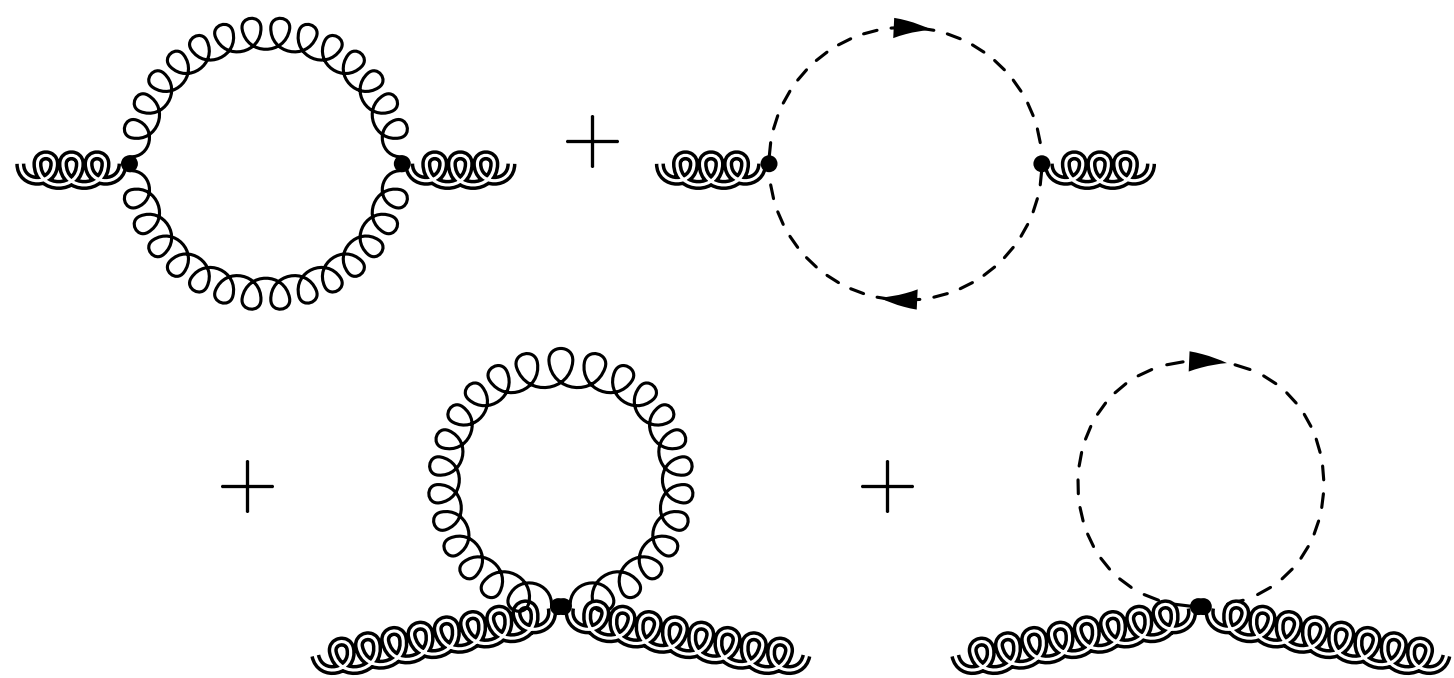


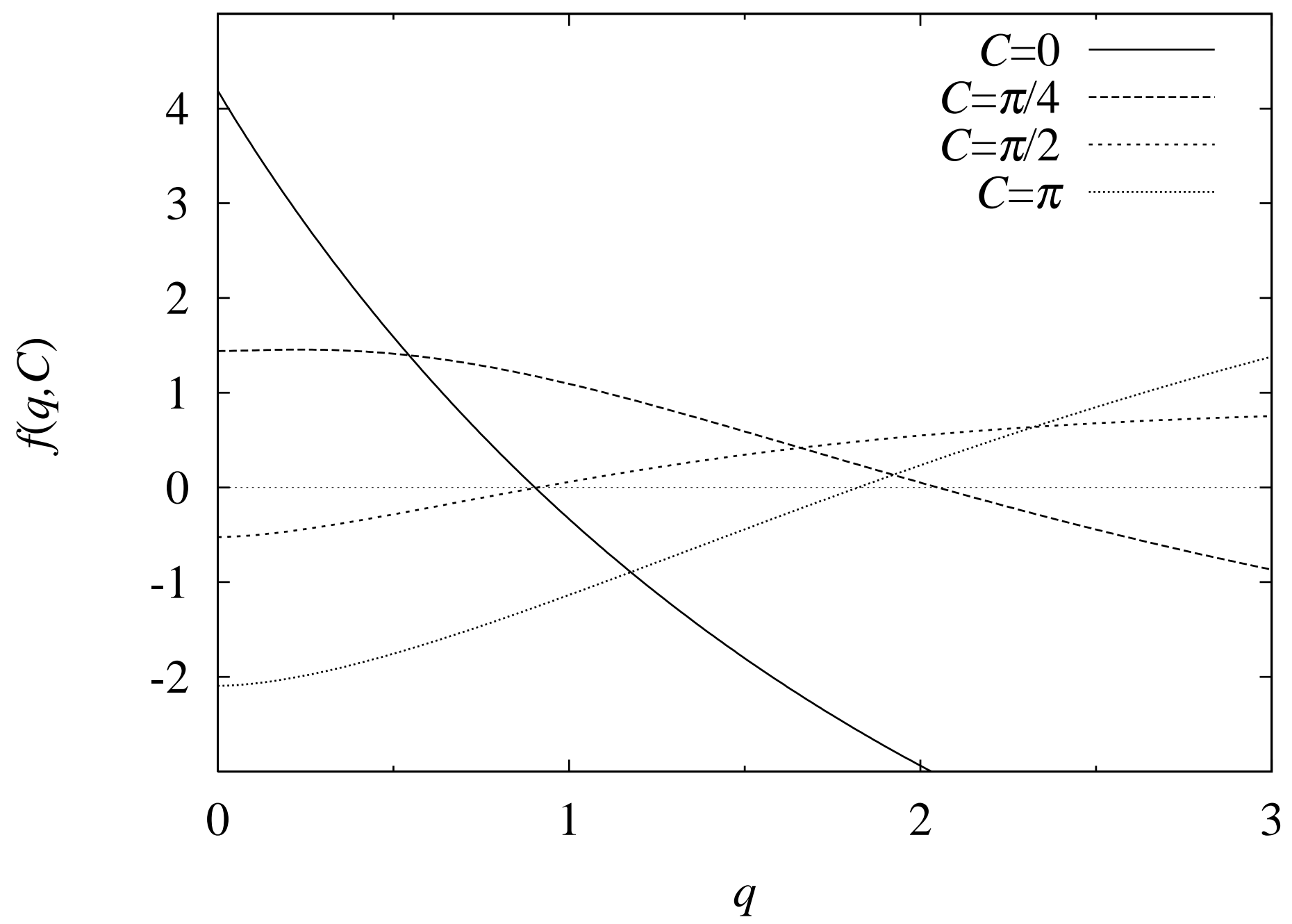

声. 


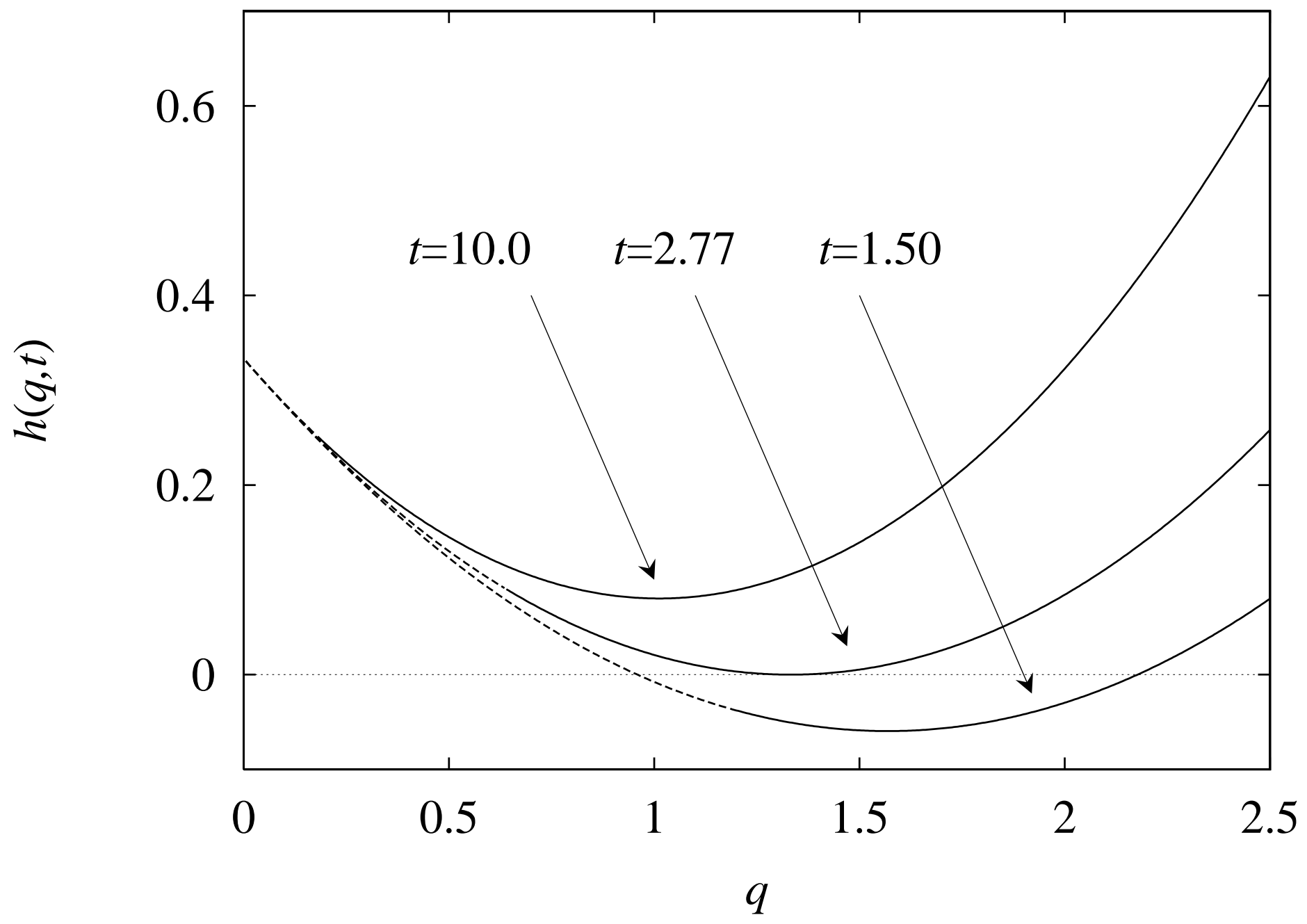

D. 
Figure 5

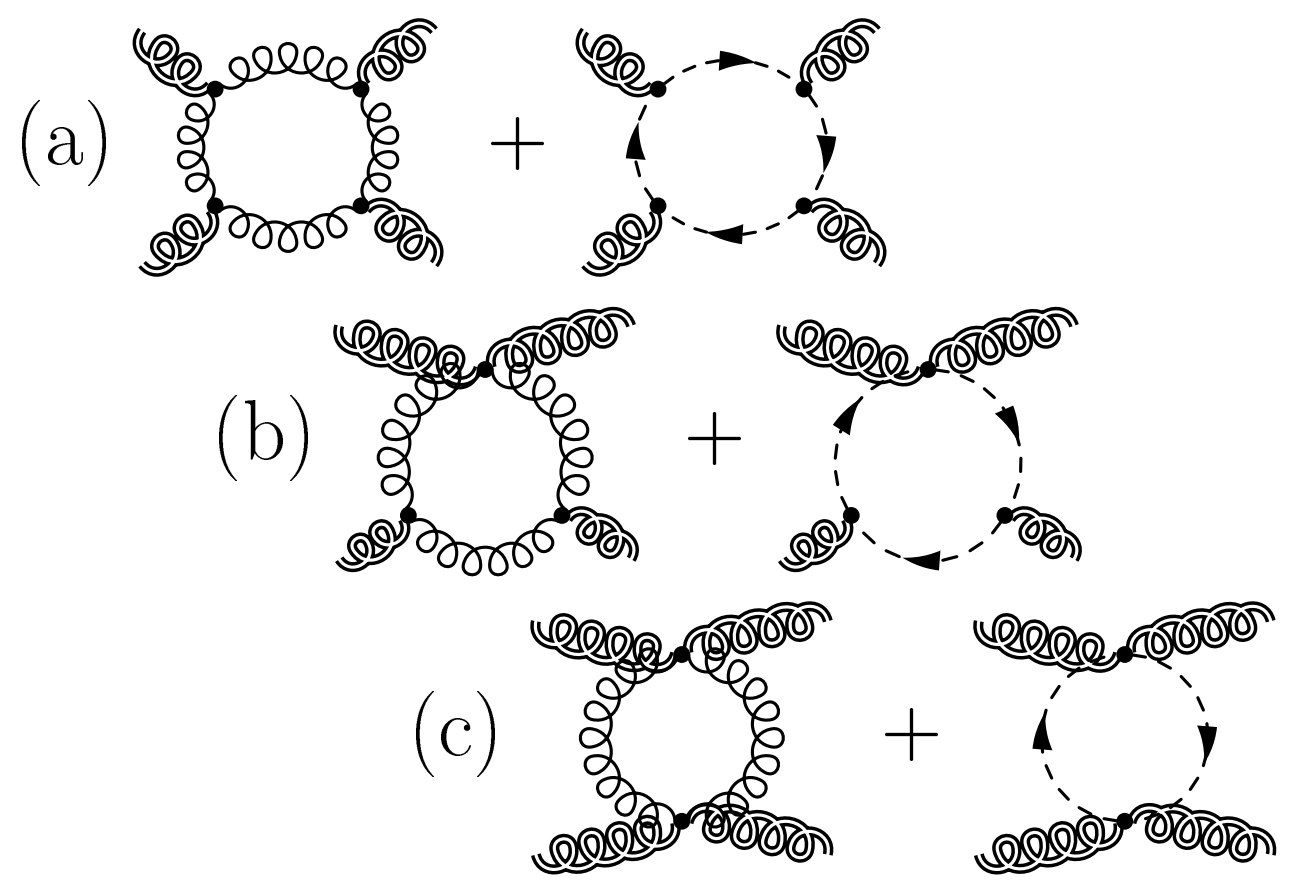

Figure 6

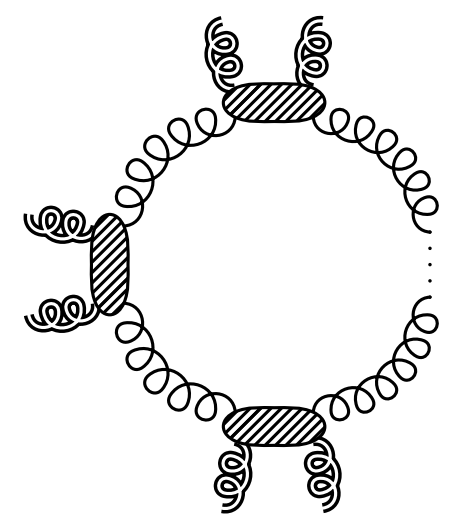




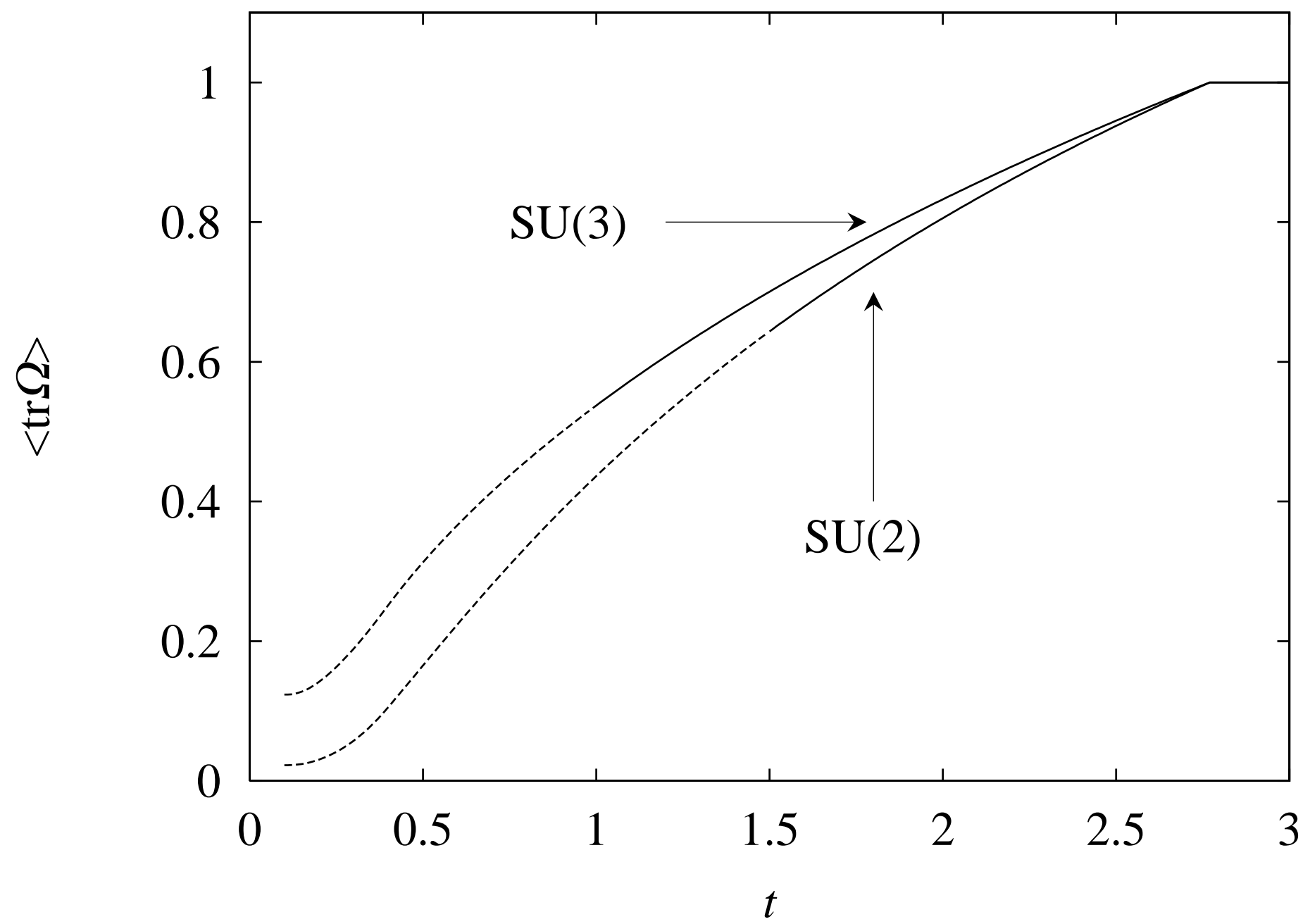

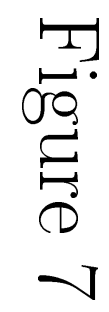




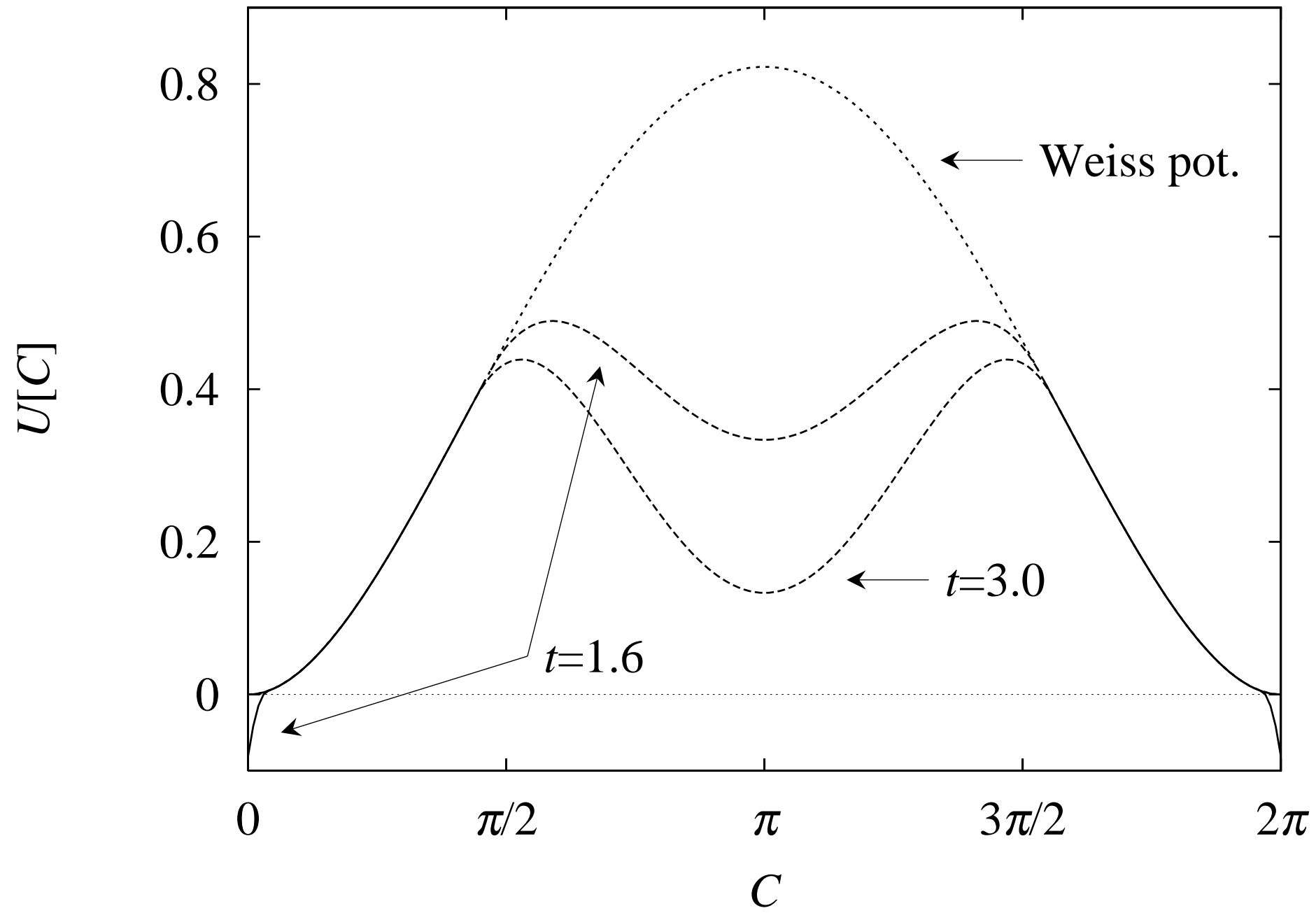

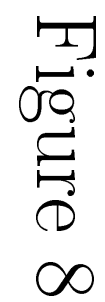

